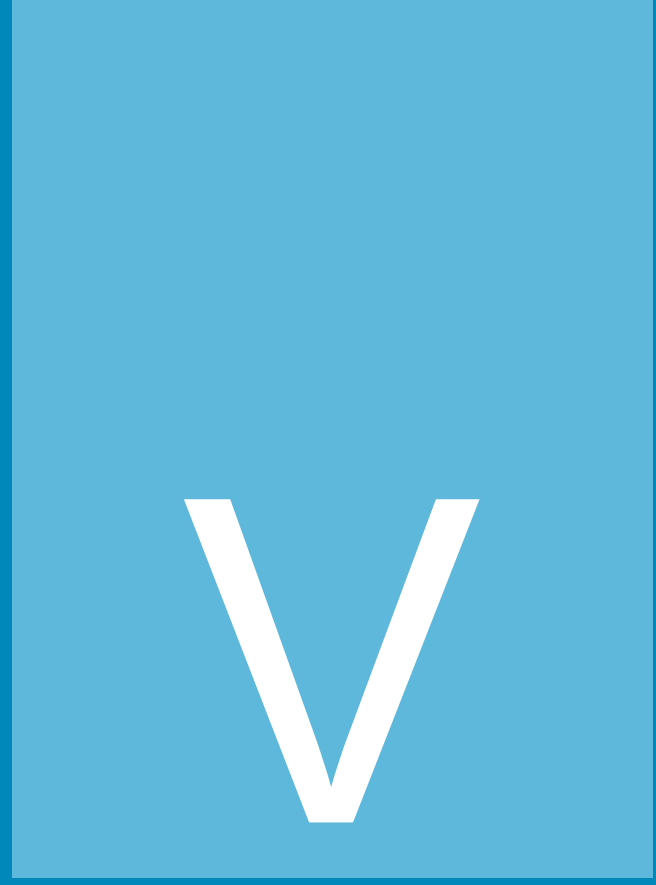

\title{
FACTORES DETERMINANTES QUE INCIDEN EN LA DESERCIÓN DE LOS ESTUDIANTES UNIVERSITARIOS
}

\section{Determinant Factors that Impact in the Defection of the University Students}

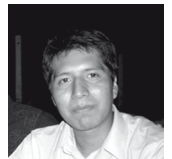

\section{Effer Apaza}

Ingeniero en Estadística por la Universidad Nacional del Altiplano. Actualmente se desempeña como docente de la Facultad de Ingeniería y Arquitectura de la Universidad Peruana Unión. Asimismo trabaja en el área de investigación de la misma universidad.

\section{Francisco Huamán}

Alumno de la Carrera de Marketing y Negocios Internacionales de la Universidad Peruana Unión. En la actualidad se desempeña como miembro del grupo de investigación de Mercado "Target" de la Universidad Peruana Unión. 


\section{Resumen}

Este artículo aborda el tema de la deserción de estudiantes en la educación superior universitaria desde una perspectiva conceptual. Se analizan las distinciones que son posibles de efectuar con respecto a este término, con el propósito de poder adoptar y entender ampliamente las definiciones para futuros trabajos sobre factores que influyen en la deserción universitaria. Además de haber recolectado una gran variedad de conceptos psicológicos, económicos, sociológicos, organizacionales y de las interacciones entre el estudiante y la institución. Dichos factores son predictivos para el abandono estudiantil, dado que habría que dar más cuidado a algunas variables que son más críticas para la toma de decisiones en los estudiantes.

Palabras clave: Deserción, estudiantes, educación universitaria.

\section{Abstract}

Through this article I address the issue of dropout students in university education from a conceptual perspective. It discusses the distinctions that are possible to make with respect to this term, in order to adopt fully understand the definitions for future work on factors influencing the college dropout. In addition to having collected a variety of concepts among psychological, economic, sociological, organizational, and interactions between the student and the institution. These factors are predictive for student dropout. Given that more care should be given to some variables that are most critical to decision making in students.

Keywords: Desertion, students, university education. 


\section{Introducción}

La deserción estudiantil es uno de los problemas que aborda la mayoría de las instituciones de educación superior de toda Latinoamérica. A través de distintas investigaciones, se percibe un número importante de estudiantes que no logran culminar sus estudios universitarios, con el consecuente costo social asociado a este fenómeno. Por lo anterior, algunos países han comenzado a diseñar profundos procesos de mejoramiento para aumentar la retención en los primeros años de estudios universitarios (UNESCO 2004).

En el Perú, de acuerdo a estimaciones basadas en estadísticas nacionales, la tasa de deserción global de pregrado, según la revista Caretas (14 de agosto de 2008, № 2040, pg 33) en el año 2008 ingresaron a la universidad 142,461 estudiantes y cerca del $36 \%$ de los alumnos no se gradúan. Según el portal de Logros: "Entre 40 y 50 mil jóvenes abandonan sus estudios universitarios cada año, lo que representa no menos de cien millones de dólares desperdiciados por los padres de familia, con la consecuente frustración que ello representa para ellos mismos y para sus hijos. De éstos, el $70 \%$ corresponde a estudiantes de universidades privadas, y la diferencia de $30 \%$ a universidades estatales".

La tendencia en el sistema universitario privado va en ascenso. El número de ingresantes aumenta considerablemente cada año y el número de egresados también; pero, a un ritmo mucho más lento, lo que hace que la brecha de la deserción se haga cada año mucho más grande. Para dentro de diez años el monto acumulado por la deserción será no menor a 2 mil cien millones de dólares.

En el sistema universitario estatal la tendencia es a disminuir; pero sigue siendo preocupante, más de 13 mil jóvenes lo hicieron para el 2006.

\section{Las principales causas que se atribuyen en el Perú a la deserción}

El aspecto económico: Los padres de familia, con la mejor intención de ofrecerles a sus hijos una buena educación, asumen un compromiso de pago de pensiones que muchas veces está fuera de su alcance. Cuando ya no es posible seguir manteniendo esta obligación, sus hijos deben abandonar la universidad, con la consecuente frustración para toda la familia y, en especial, para el joven que ve truncadas las posibilidades de su desarrollo profesional.

La falta de vocación por la carrera elegida: Otro elemento presente en esta realidad es que muchos estudiantes no tienen claro si realmente tienen vo- 
cación por lo que estudian. Muchos chicos ingresan a la universidad con la esperanza que realmente disfrutarán la profesión elegida; pero luego, sufren una profunda decepción al descubrir que lo que estudian no les agrada o no es lo que esperaban.

Esta investigación busca determinar los factores que inciden en la deserción estudiantil a través de la revisión bibliográfica.

La presente investigación es importante porque nos muestra un marco referencial sobre los factores de deserción en los estudiantes universitarios, del mismo modo nos ayuda a crear programas que aminoren el índice de deserción. Asimismo, permitirá identificar algunos factores que podrían influir en la deserción de los estudiantes de la Universidad Peruana Unión, sede Tarapoto, y con ello permitir que la universidad pueda adoptar planes que reduzcan el índice de deserción.

El número de matriculados en la Universidad Peruana Unión - Filial Tarapoto, se muestran en la siguiente tabla (Tabla 1), la cual describe como fue cambiando estos últimos tres años (Figura 1).

Tabla 1. Números de alumnos matriculados por ciclos en los últimos 3 años, UPeU.FT

\begin{tabular}{|c|c|c|c|}
\hline \multicolumn{2}{|c|}{ Año académico } & \multicolumn{2}{|c|}{ Ciclo Académico } \\
\hline Año & Frecuencia & Ciclo & Frecuencia \\
\hline \multirow[t]{2}{*}{2009} & 801 & 1 & 417 \\
\hline & & II & 384 \\
\hline \multirow[t]{2}{*}{2010} & 1005 & I & 518 \\
\hline & & II & 487 \\
\hline \multirow[t]{2}{*}{2011} & 1525 & I & 763 \\
\hline & & II & 762 \\
\hline
\end{tabular}

Fuente: UPeU-FT 


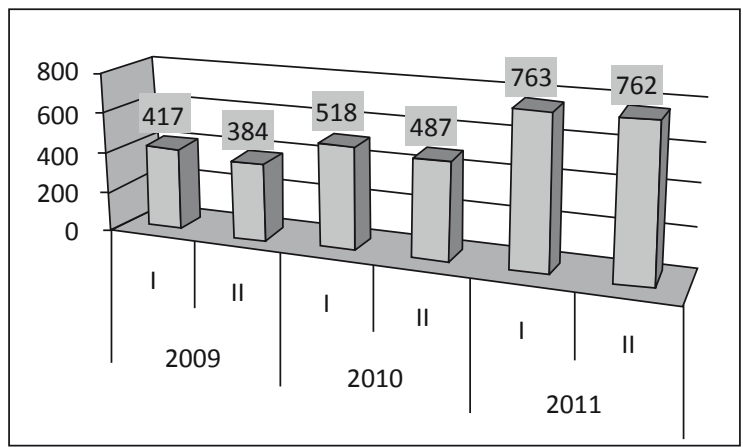

Fuente propia

Se observa que el años 2009 se obtuvo una matricula de 417 estudiantes, que para el segundo ciclo bajo a 384 estudiantes, teniendo un índice de deserción del 7,9\%.

También en el año 2010 se observa que en el primer ciclo se obtuvo una matrícula de 518 estudiantes, para el segundo ciclo del mismo año, el número total de estudiantes matriculados fue de 487. Lo cual nos da un índice de deserción del 6\% para el año 2010.

Finalmente el año (2011) el número de matriculados fue de 763 estudiantes, que para el segundo ciclo el número de estudiantes matriculados es de 762. Se nota que la deserción fue mínima, lo cual indica un 0,1\% de deserción.

\section{Revisión bibliográfica}

\section{Deserción Estudiantil}

Tinto (1989), afirma que el estudio de la deserción en la educación superior es extremadamente compleja, ya que implica no solo una variedad de perspectivas sino, además, una gama de diferentes tipos de abandono.

Según Gonzales (2005), la deserción tiene consecuencias sociales en términos de las expectativas de los estudiantes y sus familias; emocionales es por la disonancia entre las aspiraciones de los jóvenes y sus logros y, también, importante consecuencias económicas tanto para las personas como para el sistema en su conjunto.

De acuerdo a Braxton et al.(1997), los enfoques del análisis de la deserción pueden ser agrupadas en cinco grandes categorías, dependiendo 
del énfasis que otorgan las variables explicativas, ya sean individuales, institucionales o del medio familiar. De esta manera es posible reconocer los siguientes enfoques:

\section{Psicológico}

Esto señala que los rasgo de la personalidad son los que diferencia a los estudiantes que terminan sus estudios regulares de aquellos que no lo logran, cuyas bases fundamentales fueron los procesos psicológicos relacionados con la integración académica y social. Estos autores presentaron cuatro teorías psicológicas como línea de base del modelo: (a) Teoría de actitud y comportamiento, la que proviene de la estructura de su modelo; (b) Teoría del comportamiento de copia, la habilidad para entrar y adaptarse a un nuevo ambiente; (c) Teoría de autoeficacia, una percepción individual capaz de tratar con tareas y situaciones específicas; (d) Teoría de atribución, donde un individuo tiene un fuerte sentido de control interno. Estos autores enfatizaron la importancia que posee la institución al disponer de servicio de enseñanza, comunidades de aprendizaje al primer año, seminarios de orientación profesional y programas de mentoring para apoyar el éxito de los estudiantes.

\section{Económicos}

Según las investigaciones desarrolladas se pueden distinguir dos modelos: (1) Costo/Beneficio: consiste en que cuando los beneficios sociales y económicos asociados a los estudiantes son percibidos como mayores que los derivados por actividades alternas, como por ejemplo un trabajo, por lo cual el estudiante opta por permanecer en la universidad y (2) Focalización de Subsidio: consiste en la entrega de subsidios que constituyen una forma de influir sobre la deserción. Estos subsidios están dirigidos a los grupos que presentan limitaciones reales para costear sus estudios. Este modelo busca privilegiar el impacto efectivo de los beneficios estudiantiles por sobre la deserción, dejando de lado las percepciones acerca de la adecuación de dichos beneficios o el grado de ajuste de éstos a los costos de los estudios. Las ayudas proporcionadas a los estudiantes en forma de becas constituyen un factor de peso en las posibilidades de permanencia, observándose que las tasas de deserción varían dependiendo de la cantidad y duración de la ayuda financiera con la que cuenta el estudiantado e inclusive ante apuros económicos se provoca un impacto importante en el abandono temprano. 


\section{Sociológicos}

Los modelos sociológicos enfatizan respecto a la influencia en la deserción de factores externos al individuo, adicionales a los psicológicos, se basó en el modelo suicida indicando que la deserción es el resultado de la falta de integración de los estudiantes en el entorno de la educación superior; aduce que el medio familiar es una de las muchas fuentes que expone a los estudiantes a influencias, expectativas y demandas, las que a su vez afectan su nivel de integración social en la universidad; la congruencia normativa actúa directamente sobre el rendimiento académico, el desarrollo intelectual, el apoyo de pares y la integración social. Este autor señala que si las influencias señaladas no se producen en la dirección positiva, implica rendimiento académico insatisfactorio, bajo nivel de integración social y de satisfacción y compromiso institucional y una alta probabilidad de que el estudiante decida abandonar sus estudios. Este autor encontró seis predictores de la deserción estudiantil en college's norteamericanos: Integración académica, integración social, estado socioeconómico género, calidad de la carrera y el promedio de notas en cada semestre.

\section{Organizacionales}

Este modelo de análisis sostiene que la deserción depende de las cualidades de la organización en la integración social y más, particularmente, en el abandono de los estudiantes que ingresan a ella. Este enfoque es altamente relacionado a la calidad de la docencia y de la experiencia de aprender en forma activa por parte de los estudiantes en las aulas, las cuales afectan positivamente la integración social del estudiante (Braxton et al. 1997).

\section{De Interacciones}

Tinto (1975), explica el proceso de permanencia en la educación superior como una función del grado de ajuste entre el estudiante y la institución, adquirido a partir de las experiencias académicas y sociales (integración). Este autor expande el modelo, incorporando la teoría de intercambio. La base de la teoría de intercambio se encuentra en el principio de que los seres humanos evitan las conductas que implican un costo de algún tipo para ellos y buscan recompensas en las relaciones, interacciones y estados emocionales. El mismo autor afirma que los estudiantes actúan de acuerdo con la teoría de intercambio en la construcción de su integración social y académi- 
ca, es decir, si el estudiante percibe que los beneficios de permanecer en la universidad son mayores que los costos personales, entonces el estudiante permanecerá en la institución.

En el sentido contrario, si percibe que otras actividades son percibidas como fuentes de recompensas más altas, el estudiante tenderá a desertar. Tinto (1987), sugiere que una buena integración es uno de los aspectos más importantes para la permanencia, y que esta integración depende de: las experiencias durante la permanencia en la universidad, las experiencias previas al acceso universitario y las características individuales que, por otro lado, son susceptibles a las políticas y prácticas universitarias.

Posteriormente, Tinto $(1989,1993)$, profundiza el análisis del modelo inicial del año 1975 y en esta oportunidad se observa que a medida que el estudiante avanza en su trayectoria académica, diversas variables contribuyen a reforzar su adaptación a la institución que seleccionó, ya que ingresa a ella con un conjunto de características que influyen sobre su experiencia, en la educación terciaria. Estas características comprenden antecedentes familiares, tales como los valores que ésta sustenta, además de sus atributos personales y de la experiencia académica preuniversitaria. Todos estos aspectos relevantes se combinan para influir sobre el compromiso inicial con la institución, así como para la consecución de su meta, que es la graduación o titulación. Los estudios que han empleado el modelo de Tinto confirman la capacidad predictiva directa de la integración social sobre la deserción, sobre todo en las universidades tradicionales. Sin embargo, algunos estudios han puesto en duda si el modelo del Tinto es aplicable a todos los estudiantes e instituciones de educación superior.

Estos estudios indican que si bien la integración social y académica son factores importantes para predecir la persistencia, no tienen la misma importancia en todos los estudiantes, reportaron que la integración académica es más fuerte que los compromisos o metas institucionales; por tanto, los compromisos institucionales tienen un efecto indirecto sobre la permanencia a través de la integración social, las características del modelo de productividad desarrollado en el contexto de las organizaciones laborales. Asimismo sostiene que inciden en la deserción los siguientes factores:(1) Factores Académicos: preuniversitarios, integración académica y desempeño académico;(2) Factores Psicosociales: metas, utilidad percibida, interacción con pares y docentes; (3) Factores Ambientales: financiamiento, oportunidad de transferirse, relaciones sociales externas;(4) Factores de Socialización: rendimiento académico, adaptación y compromiso institucional.

En estudios más recientes se diseñaron modelos para explicar el proceso de la persistencia en los college's norteamericanos que se basa, predominantemente, en factores externos a la institución. Las variables consi- 
deradas se relacionan con la institución, la persona o el ambiente; es decir, el ámbito exterior a la institución. Consideraron que estos factores también tienen un peso significativo en la deserción, en particular en la deserción voluntaria.

Vásquez (1997), realizó una investigación sobre factores biológicos, socioeconómicos y culturales que influyen en la deserción en estudiantes de enfermería de la Universidad Nacional de Trujillo. De otro lado, el medio familiar puede jugar un rol importante para que el estudiante termine su carrera o la abandone; pero, también puede ser el medio universitario mismo. Igualmente, un hogar pobre donde el nivel educativo de los padres es bajo, tendría poca influencia para que el estudiante se mantenga en sus estudios. Este fenómeno es preocupante en estudiantes de las ciencias de la salud, particularmente en facultades o escuelas de enfermería del Perú.

Latiesa (1992), señala que la deserción universitaria es uno de los procesos de selección que se opera en la enseñanza superior, una medida del rendimiento académico del alumnado y de la eficacia del Sistema Educativo en general. Esta precisión comprende tres términos: "proceso de selección", "medida del rendimiento académico", y "eficacia del sistema educativo". Algunos investigadores ensayan el abordaje de tres dimensiones: el éxito en los estudios, el retraso y el abandono de los mismos. Aquí el debate se abre nuevamente ya que las experiencias según las colecciones de datos no son necesariamente convergentes. En primer lugar porque las universidades asumen con cierta 'naturalidad' que la deserción o abandono es uno de los resultados posibles, a diferencia de otras instituciones educativas en las que la cobertura de grupos de edades de niños y jóvenes es parte constitutiva del objetivo de la formación (primaria y secundaria), o de aquellas instituciones de formación de oficiales civiles o militares en las que el abandono tiene que ser mínimo por razones propias al fin mismo de la institución.

\section{Conclusiones}

1. La deserción de los estudiantes es el resultado de la combinación y efectos de distintas variables, las cuales muchas veces son independientes y no se pueden controlar.

2. El estudiante mantendrá una condición de equilibrio (permanencia) al combinar los factores psicológicos, académicos, sociales e institucionales en constante fortalecimiento de uno u otro factor. 
3. Si uno de los factores se debilita, se recomienda mantener al estudiante en equilibrio, es necesario que los otros factores sean capaces de reaccionar para suplir los aspectos necesarios para mantener el equilibrio inicial.

\author{
Effer Apaza \\ Universidad Peruana Unión \\ email: edi_efr@msn.com \\ Francisco Huamán \\ Universidad Peruana Unión \\ email: xavier_0428@hotmail.com \\ Recibido: 12 de Marzo de 2012 \\ Aceptado: 16 de Mayo de 2012
}

\title{
Referencias
}

Braxton, J., A. Sullivan and R. Johnson Jr. (1997). Appraising Tinto's theory of college student departure. In: J.C. Smart (Ed.), Higher education: Handbook of theory and research. New York. USA. pp. 107-164.

González, L. (2005). Estudio sobre la repitencia y deserción en la educación superior chilena. Digital Observatory for higher education in Latin America and The Caribbean. IESALC UNESCO

Latiesa, M. (1992). La deserción universitaria. Madrid: CIS siglo XXI .

Tinto, V. (1989). Definir la deserción: una cuestión de perspectiva. Revista de Educación Superior N 71, ANUIES, México.

Vásquez C (1993). Algunos factores biológicos, socioeconómicos y culturales influyentes en la deserción de los estudiantes de enfermería. Tesis de Maestría no publicada. Trujillo (Perú): Universidad Nacional de la Libertad. 1993. 